\title{
Cryptographic Protocols from Lattices
}

\author{
Eike Kiltz \\ CWI, Amsterdam \\ kiltz@cwi.nl
}

\begin{abstract}
In this talk, we will introduce abstract tools and techniques for working with lattices from the perspective of a cryptographic protocol designer. We will also show how to apply these tools to yield a number of provably secure public-key primitives ranging from digital signatures and public-key encryption, to more advanced protocols such as (hierarchical) identity-based encryption.
\end{abstract}

